\title{
Toward harmonized phenotyping of human myeloid-derived suppressor cells by flow cytometry: results from an interim study
}

\author{
Susanna Mandruzzato ${ }^{1,2}$ - Sven Brandau ${ }^{3}$ - Cedrik M. Britten ${ }^{4,5}$. \\ Vincenzo Bronte $^{6}$ - Vera Damuzzo ${ }^{1,2}$ - Cécile Gouttefangeas ${ }^{7}$ - Dominik Maurer ${ }^{8}$. \\ Christian Ottensmeier $^{9}$ Sjoerd H. van der Burg ${ }^{10} \cdot$ Marij J. P. Welters $^{10}$ • \\ Steffen Walter ${ }^{8}$
}

Received: 4 September 2015 / Accepted: 12 December 2015 / Published online: 4 January 2016

(C) The Author(s) 2015. This article is published with open access at Springerlink.com

\begin{abstract}
There is an increasing interest for monitoring circulating myeloid-derived suppressor cells (MDSCs) in cancer patients, but there are also divergences in their phenotypic definition. To overcome this obstacle, the Cancer Immunoguiding Program under the umbrella of the Association of Cancer Immunotherapy is coordinating a proficiency panel program that aims at harmonizing MDSC phenotyping. After a consultation period, a two-stage approach was designed to harmonize MDSC phenotype. In the first step, an international consortium of 23 laboratories immunophenotyped 10 putative MDSC subsets on pretested, peripheral blood mononuclear cells of healthy donors to assess the level of concordance and define robust marker combinations for the identification of circulating MDSCs. At this stage, no mandatory requirements to standardize reagents or protocols were introduced. Data analysis revealed a small intra-laboratory, but very high
\end{abstract}

Electronic supplementary material The online version of this article (doi:10.1007/s00262-015-1782-5) contains supplementary material, which is available to authorized users.

Susanna Mandruzzato

susanna.mandruzzato@unipd.it

1 Section of Oncology and Immunology, Department of Surgery, Oncology and Gastroenterology, University of Padova, Via Gattamelata, 64, 35128 Padua, Italy

2 Veneto Institute of Oncology IOV - IRCCS, Padua, Italy

3 Department of Otorhinolaryngology, University Hospital Essen, Essen, Germany

4 TRON Translationale Onkologie an der Universitätsmedizin der Johannes Gutenberg-Universität Mainz GmbH, Mainz, Germany

5 Present Address: Cell Therapy Group, Immuno-Oncology and Combinations, GlaxoSmithKline, Stevenage, UK inter-laboratory variance for all MDSC subsets, especially for the granulocytic subsets. In particular, the use of a deadcell marker altered significantly the reported percentage of granulocytic MDSCs, confirming that these cells are especially sensitive to cryopreservation and/or thawing. Importantly, the gating strategy was heterogeneous and associated with high inter-center variance. Overall, our results document the high variability in MDSC phenotyping in the multicenter setting if no harmonization/standardization measures are applied. Although the observed variability depended on a number of identified parameters, the main parameter associated with variation was the gating strategy. Based on these findings, we propose further efforts to harmonize marker combinations and gating parameters to identify strategies for a robust enumeration of MDSC subsets.

Keywords Myeloid-derived suppressor cells . Phenotyping · Proficiency panel · Flow cytometry

6 Section of Immunology, Department of Pathology and Diagnostics, Verona University Hospital, Verona, Italy

7 Department of Immunology, Institute for Cell Biology, University of Tübingen, Tübingen, Germany

8 Immatics Biotechnologies GmbH, Tübingen, Germany

9 Cancer Sciences Unit, Faculty of Medicine, Experimental Cancer Medicine Centre, Southampton General Hospital, University of Southampton, Tremona Road, Southampton, UK

10 Department of Clinical Oncology, Leiden University Medical Center, Leiden, The Netherlands 


$\begin{array}{ll}\text { Abbreviations } \\ \text { CIMT } & \text { Association for Cancer Immunotherapy } \\ \text { CIP } & \text { CIMT Immunoguiding Program } \\ \text { DCM } & \text { Dead-cell marker } \\ \text { G-MDSC } & \text { Granulocytic MDSC } \\ \text { i-MDSC } & \text { Immature MDSC } \\ \text { M-MDSC } & \text { Monocytic MDSC } \\ n \% \text { MDSC } & \text { Normalized MDSC frequencies }\end{array}$

\section{Introduction}

Myeloid-derived suppressor cells (MDSCs) are a heterogeneous group of myeloid cells at different stages of differentiation that are often expanded in cancer patients and capable to suppress immune responses [1]. In recent years, recognition of the clinical relevance of MDSCs has steadily increased. MDSC levels correlate negatively with prognosis and overall survival in cancer patients, and the accumulation of this population promotes tumor progression and is linked to tumor stage and worse prognosis of cancer patients [2-5].

MDSCs were originally described in mice, on the basis of the co-expression of CD11b and Gr-1. However, subsequent studies elucidated that $\mathrm{CD} 11 \mathrm{~b}^{+} / \mathrm{Gr}-1^{+}$cells are not a homogeneous cell population, but rather a heterogeneous group of myeloid cells, endowed with different suppressive ability. At present, it is well known that there are at least two main subsets of MDSCs present in tumorbearing mice, the monocytic (M-MDSC) and the granulocytic (G-MDSC) ones and that these cell populations can be properly identified by using a set of accessory markers. In particular, the differential expression of Ly6C and Ly6G, the two isoforms of Gr-1, allows the identification of G-MDSC as $\mathrm{CD}_{11} \mathrm{~b}^{+} / \mathrm{Gr}-1^{\mathrm{high}} / \mathrm{Ly} 6 \mathrm{C}^{-} / \mathrm{Ly} 6 \mathrm{G}^{\text {high }}$ and M-MDSC as CD $11 b^{+} / \mathrm{Gr}-1^{\text {int }} / \mathrm{Ly}^{-} \mathrm{C}^{\text {high }} / \mathrm{Ly} 6 \mathrm{G}^{-}$[6].

The phenotyping of human MDSCs is complicated by the fact that Gr-1 is not expressed on human leukocytes. Thus, multiple human MDSC subsets with different phenotypes have been documented in several types of tumors in the last 20 years (reviewed in [7]). All reported phenotypes are defined on the basis of a combination of myeloid markers and suffer from the lack of a specific marker. The initial assumption that MDSCs are solely constituted of immature myeloid cells [8-10] is now challenged by reports describing MDSCs with morphology and phenotype consistent with those of cells with more differentiated features. At present, it appears that all MDSC phenotypes can be allocated to one of the at least three main subsets, of which each contains more than one cell population. M-MDSCs share the morphology of monocytes and are characterized by the expression of CD14 and lack of CD15 [11, 12]; G-MDSCs contain a cell population resembling granulocytes and are phenotypically characterized by CD15 and CD66b expression and the absence of CD14 [12-16]; immature MDSCs (i-MDSC) are cells with immaturity characteristics, defined essentially as lineage negative cells [3, 17].

Although there is an increasing relevance of MDSCs for tumor immunology, there is lack of a uniformly used and broadly accepted classification of human MDSCs (in terms of both subsets types and identification markers). This motivated the design of a proficiency panel by the CIMT (Association for Cancer Immunotherapy) Immunoguiding Program (CIP) (http://www.cimt.eu/workgroups/cip/) to assess the heterogeneity in current human MDSC phenotyping approaches and harmonize them across different laboratories. The first step of the proficiency panel, which is the focus of this communication, did not include a functional assay, since the suppressive activity of most of these subsets was already reported [7]; hence, this study was mainly designed to assess intra-laboratory and inter-laboratory variance and to define a robust marker combination for the phenotypical identification of circulating MDSCs. The parameters associated with high variance identified from this first phase will be harmonized in the second step with the aim to decrease the variability of MDSC quantification.

\section{Materials and methods}

\section{Healthy donors}

After given written informed consent, leukapheresis was performed for 23 healthy individuals. Peripheral blood mononuclear cells (PBMCs) were centrally prepared by Ficoll gradient and cryopreserved as described previously [4]. The healthy donors were screened by flow cytometry for the presence of the 10 putative MDSC subsets by the organizing committee, and three representative donors with the most significant expansion of these subsets were selected for the first phase of the proficiency panel.

\section{Proficiency panel}

Two vials of each donor were shipped to the participant laboratories in dry ice and stored in liquid nitrogen upon arrival. The participating laboratories were asked to perform the immunophenotyping of 10 putative subsets of MDSCs on the cryopreserved samples from the three selected healthy donors (HBC-480, HBC-514, L29-3). Experiment guidelines to indicate the mandatory parameters to be fulfilled and with exemplary staining cocktails, protocol and gating strategy (Fig. 1) were provided to the participants. The proposed staining cocktails contain 7 markers commonly used for MDSC recognition (as deduced from two webinars held on MDSCs by CIP) plus a 
dead-cell marker (DCM): HLA-DR, CD14, CD15, CD11b, CD33, lineage cocktail (defined as CD3/14/19/56) and CD124. This marker combination allows the identification of 10 putative MDSC subsets: MDSC1 $\mathrm{CD} 14^{+} / \mathrm{CD} 124^{+}$ [12]), MDSC2 CD15 $/ \mathrm{CD} 124^{+}$[12], MDSC3 Lin ${ }^{-} / \mathrm{HLA}-$ $\mathrm{DR}^{-} / \mathrm{CD} 33^{+}[10], \mathrm{MDSC} 4 \mathrm{CD} 14^{+} / \mathrm{HLA}^{+} \mathrm{DR}^{\text {low/- }}[11]$, MDSC5 CD $15^{+} / \mathrm{CD} 14^{-} / \mathrm{CD}_{11 b^{+}}[15], \mathrm{MDSC} \mathrm{CD} 15^{+} /$ $\mathrm{FSC}^{\text {low }} / \mathrm{SSC}^{\text {high }}$ [13], MDSC7 $\mathrm{CD} 15^{-} / \mathrm{CD} 14^{+} / \mathrm{CD} 33^{\text {high} /}$ HLA-DR $^{\text {low }}$ [18], MDSC8 $\mathrm{CD}^{2} 5^{+} / \mathrm{CD} 33^{\text {high }}$, MDSC9 $\mathrm{CD} 14^{-} / \mathrm{CD} 15^{-} / \mathrm{CD} 33^{\text {high }}$ and MDSC10 $\mathrm{Lin}^{-} / \mathrm{HLA}^{-\mathrm{DR}^{\text {low }} /}$ $\mathrm{CD}_{11} \mathrm{~b}^{+}$[19]. Participants were asked to perform the staining twice, in two separate days, in order to calculate intralaboratory variance. For each donor in each of the two experimental runs, participants were asked to determine cell viability after thawing, test and report the number of total cells, singlets, monocytes and lymphocytes plus 10 putative MDSC phenotypes using one 8-color panel and/or three 4-color antibody flow cytometry panels.

In addition, guidelines indicated to use at least one million PBMCs per test, not to perform a resting period after thawing the cells and not to fix the stained cells.

Participants were asked to analyze the flow data using their own gating strategy and to report it in single layouts; moreover, they were asked to determine the absolute number of the requested cell populations with or without the presence of a DCM.

\section{Central data analysis}

Results from the 23 participating laboratories were centrally collected and analyzed. Absolute numbers of the 10 putative MDSC subsets were normalized to the count of lymphocytes + monocytes. Variance was calculated as follows: $\% \mathrm{CV}=($ standard deviation/mean $) * 100$. Comparison between $\% \mathrm{CV}$ or normalized frequencies of myeloid subsets identified by different groups was performed using the Wilcoxon signed-rank test. Significance level was set for $P<0.05$.

\section{Results}

To start this project, we conducted a survey by sending out a questionnaire to groups that the organizers identified as having published at least one paper on human MDSCs. We explored their interest in participating to a harmonization process for the phenotyping of human MDSCs. Results of 89 analyzed questionnaires were discussed at the CIMT Annual Meeting 2012 and in two webinars hold in September 2012 with more than 50 participants. Most research groups expressed strong interest to join this activity and, in particular, to reach an agreement for the markers required to phenotype MDSCs. From the initial survey, it appeared that overall 32 markers were used to define MDSCs, but only a core set of 7 markers was commonly used by all groups.

On the basis of these results, a consensus could be reached among the webinar participants to define the following 7 markers describing 10 different putative subsets of MDSCs for further testing: MDSC $1 \mathrm{CD} 14^{+} / \mathrm{CD} 124^{+}$ [12], MDSC2 $\mathrm{CD}^{+} 5^{+} / \mathrm{CD} 124^{+}[12]$, MDSC $3 \mathrm{Lin}^{-} / \mathrm{HLA}-$ $\mathrm{DR}^{-} / \mathrm{CD}_{3} 3^{+}[10,20]$, MDSC4 CD14 ${ }^{+} / \mathrm{HLA}^{-D R}{ }^{\text {low/- }}[11]$, MDSC5 CD15 ${ }^{+} / \mathrm{CD} 14^{-} / \mathrm{CD}_{11 \mathrm{~b}^{+}}[15], \mathrm{MDSC}^{\mathrm{CD}} 15^{+} /$ $\mathrm{FSC}^{\text {low }} / \mathrm{SSC}^{\text {high }}[13], \mathrm{MDSC} 7 \mathrm{CD} 15^{-} / \mathrm{CD} 14^{+} / \mathrm{CD} 33^{\text {high}} /$ HLA-DR $^{\text {low/- }}$ [18], MDSC8 $\mathrm{CD} 15^{+} / \mathrm{CD} 33^{\text {high }}$, MDSC9 $\mathrm{CD} 14^{-} / \mathrm{CD} 15^{-} / \mathrm{CD} 33^{\text {high }}$ and MDSC10 $\mathrm{Lin}^{-} / \mathrm{HLA}-$ $\mathrm{DR}^{\text {low/-}} / \mathrm{CD} 11 \mathrm{~b}^{+}[19]$. Lineage (Lin) was defined by the cocktail of anti-CD3/14/19/56 antibodies.

For ethical and practical reasons, it was not feasible to obtain leukaphereses from cancer patients, and therefore, PBMCs of healthy donors were used for this harmonization panel. Participants were asked to phenotype the samples for the 10 predefined putative MDSC subsets. A total of 23 laboratories (15 European and 8 from the USA) participated to the proficiency panel and reported their results to the organizing committee.

Participants were asked to stain the PBMC with antibodies against HLA-DR, CD14, CD15, CD11b, CD33, lineage cocktail (defined as specified above) and CD124 plus a dead-cell marker (DCM). The participating laboratories chose gating strategy, antibody clones and fluorochromes, protocol for staining, negative controls and the inclusion of additional markers. An exemplary, but optional, gating strategy was provided in the guidelines (Fig. 1). We mandated that centers would use either one 8-color antibody panel or three 4-color antibody panels to combine the 8 proposed markers and to perform two experimental runs for each donor on different days. As readout, participants reported the number of total events, lymphocytes, monocytes and MDSC subsets in either the presence or absence of DCM.

For each MDSC subset and each donor, we calculated the intra-assay and inter-assay variance $(\% \mathrm{CV})$ of each reported MDSC subset frequency.

Intra-laboratory variance was as expected for multicolor phenotyping assays within a lower range (range 20-45\%, Fig. 2a). However, data analysis revealed large inter-laboratory variance among the 10 MDSC subsets analyzed, ranging from 50 to $300 \%$, with the highest variance among the granulocytic subsets compared to the immature and monocytic subsets (Fig. 2b). When analyzing the influence of data normalization, we found that normalizing MDSC frequencies ( $n \%$ MDSC) to either lymphocytes or lymphocytes + monocytes generally reduced variance (Fig. 2c). Exemplary data of individual laboratories for three subsets are shown in Fig. 3 (panels a, b and c), and the descriptive statistics of the results is reported in Supplementary Table 1. 


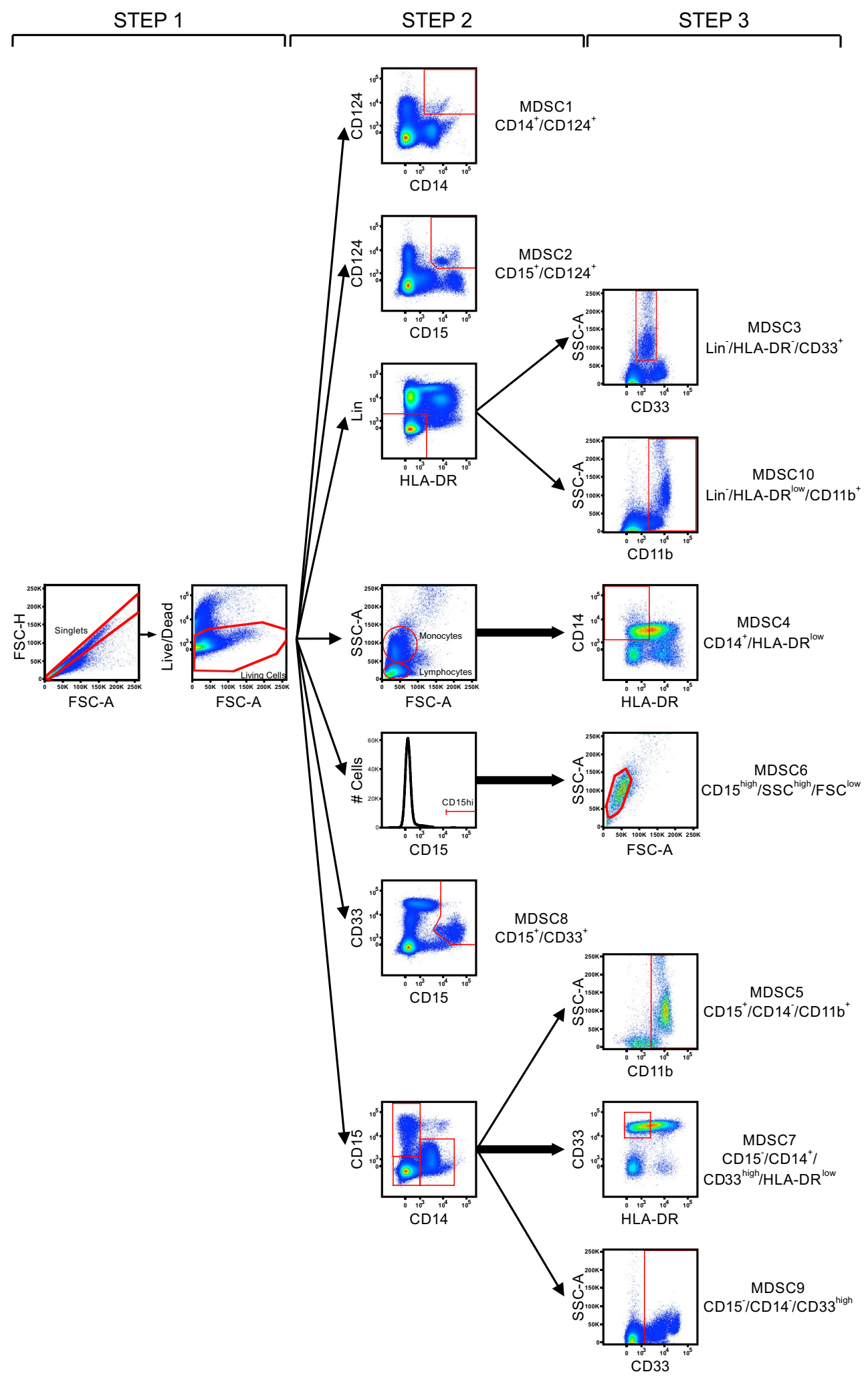

Fig. 1 Exemplary gating strategy included in the panel's guideline. The figure shows the gating strategy proposed in the guidelines and made of three sequential steps, allowing the identification of the 10 putative MDSC subsets. Step 1 consists in doublets exclusion fol- lowed by dead-cell exclusion. Step 2 allows the identification of three MDSC subsets (MDSC1-2-8), and from a consecutive gate, the other seven defined MDSC populations are identified in step 3 
In view of the large spread of the data, we set out to identify the potential sources of variability in the phenotyping of the 10 MDSC subsets. Accordingly, we analyzed the $n \%$ MDSC and the \%CV obtained from the analysis performed with or without DCM. Results indicate that the use of DCM decreases the $n \%$ MDSC of G-MDSC (MDSC2, 5, 6 and 8), M-MDSC MDSC1 and i-MDSC (MDSC 3 and 10) but not of monocytic subsets MDSC4 and 7 (Fig. 4a). The explanation for these results probably lies on the fragile nature of granulocytic cells, which are more prone to cell death during the cryopreservation and/or thawing procedure than either monocytic subsets or lymphocytes [6, 21]. The use of a dead-cell marker did not significantly affect the variance of results obtained from two tests performed by the same laboratory (intra-laboratory; Fig. 2a), while it increased the inter-laboratory variance of results (Fig. 2b; $P<0.0001$, Wilcoxon matched-pair test).

Other parameters, such as antibody clones and the use of 8-color versus 4-color panels, were difficult to analyze, because the large number of subgroups identified for each variable led to numbers too small for statistical approaches. However, results suggest that the choice of negative controls, CD124 clone and composition of the lineage cocktail were associated with high inter-laboratory variation (data not shown).

It is well known that one of the sources of inter-laboratory variability arises from manual gating analysis of flow cytometry data [22-24], and, indeed, we observed a large variability in the gating strategy developed by the participating groups. For example, some participants put the gate for $\mathrm{CD} 124^{+}$cells only on highly fluorescent cells, while others gated positive cells including also dimly fluorescent cells; as a consequence, total \% CV for MDSC1 is twofold higher than the \% CV calculated within each of the two groups that use a similar gating strategy to identify $\mathrm{CD}_{124^{+}}$cells (data not shown). Also the gating of CD33 positive cells was differently performed including CD33 low and/or high cells, thereby increasing the variance. To dissect the influence of gating analysis on the result variance, we grouped the laboratories that followed the gating example provided in our guidelines (Fig. 1) for the identification of the 10 MDSC subsets. We observed that for those participant results following a homogeneous gating approach, the $\% \mathrm{CV}$ was reduced $(P=0.0012$, Wilcoxon matched-pair test) for most MDSC subsets, especially for G-MDSCs (Fig. 4b).

\section{Discussion}

Circulating levels of MDSCs have been correlated with tumor burden and overall survival in different types of cancer, and some studies demonstrated their prognostic
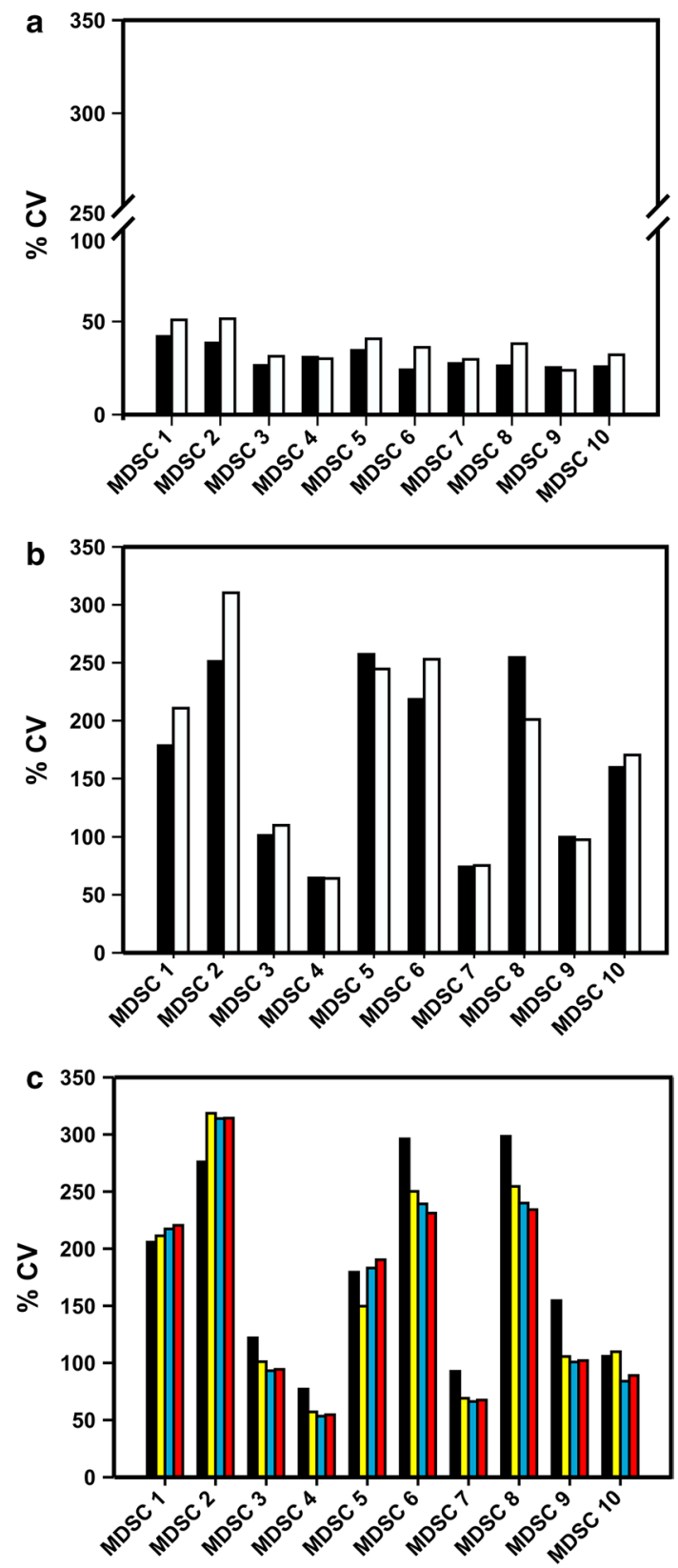

Fig. 2 Analysis of the impact of a dead-cell marker on MDSC quantification. Black bars indicate average \%CV from analyses performed without exclusion of dead cells, while white bars refer to analyses considering only live cells $(n=18$ evaluable laboratories reporting data for the 8-color panel). Intra-laboratory variance is shown in panel a, while panel b reports inter-laboratory variance. c Average $\% \mathrm{CV}$ ( $n=14$ evaluable laboratories reporting data for the 8-color panel) of the frequencies of the ten MDSC subsets without normalization (black bar) and normalized on the number of singlets (yellow bar), lymphocytes (blue bar) and lymphocytes + monocytes (red bar)

role for the outcomes of different chemotherapeutic regimens (reviewed in [7]). Therefore, it is a promising biomarker now also for response to immunotherapy. However, 
Fig. 3 Values of a representative subsets of monocytic (MDSC4 - panel a), granulocytic (MDSC5 - panel b) and immature (MDSC10 - panel c) MDSCs are shown as examples. Frequencies were normalized on the count of lymphocytes + monocytes for each laboratory performing either one 8-color panel or three 4-color panels for MDSC identification; the two paired histograms refer to the two independent experimental runs performed by each laboratory (black bars $=$ first run, gray bars $=$ second run). Missing values, not reported by single laboratories, were labeled with " $m$ "

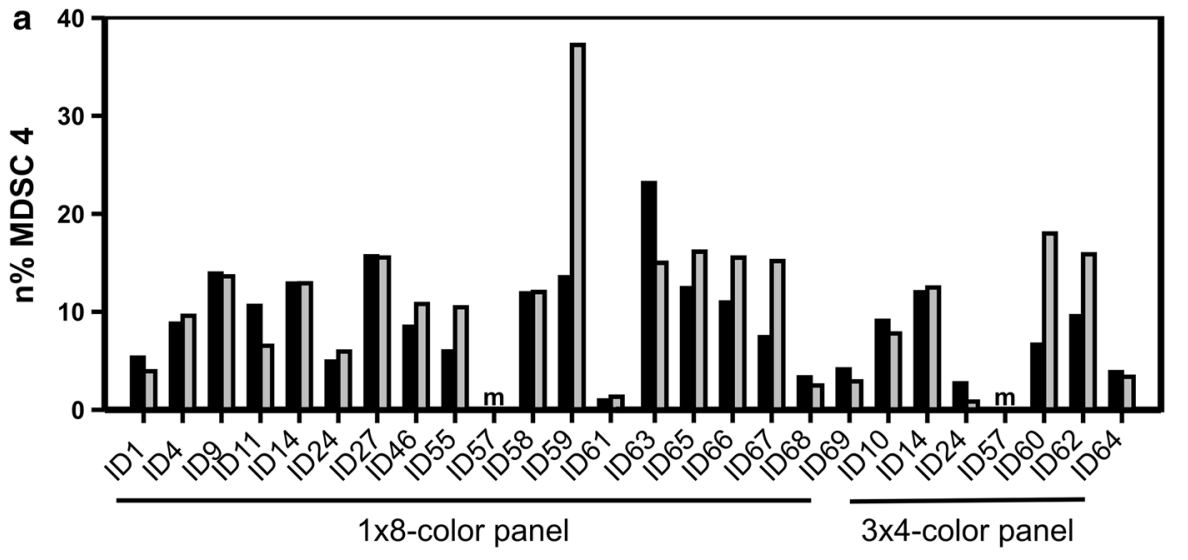

b
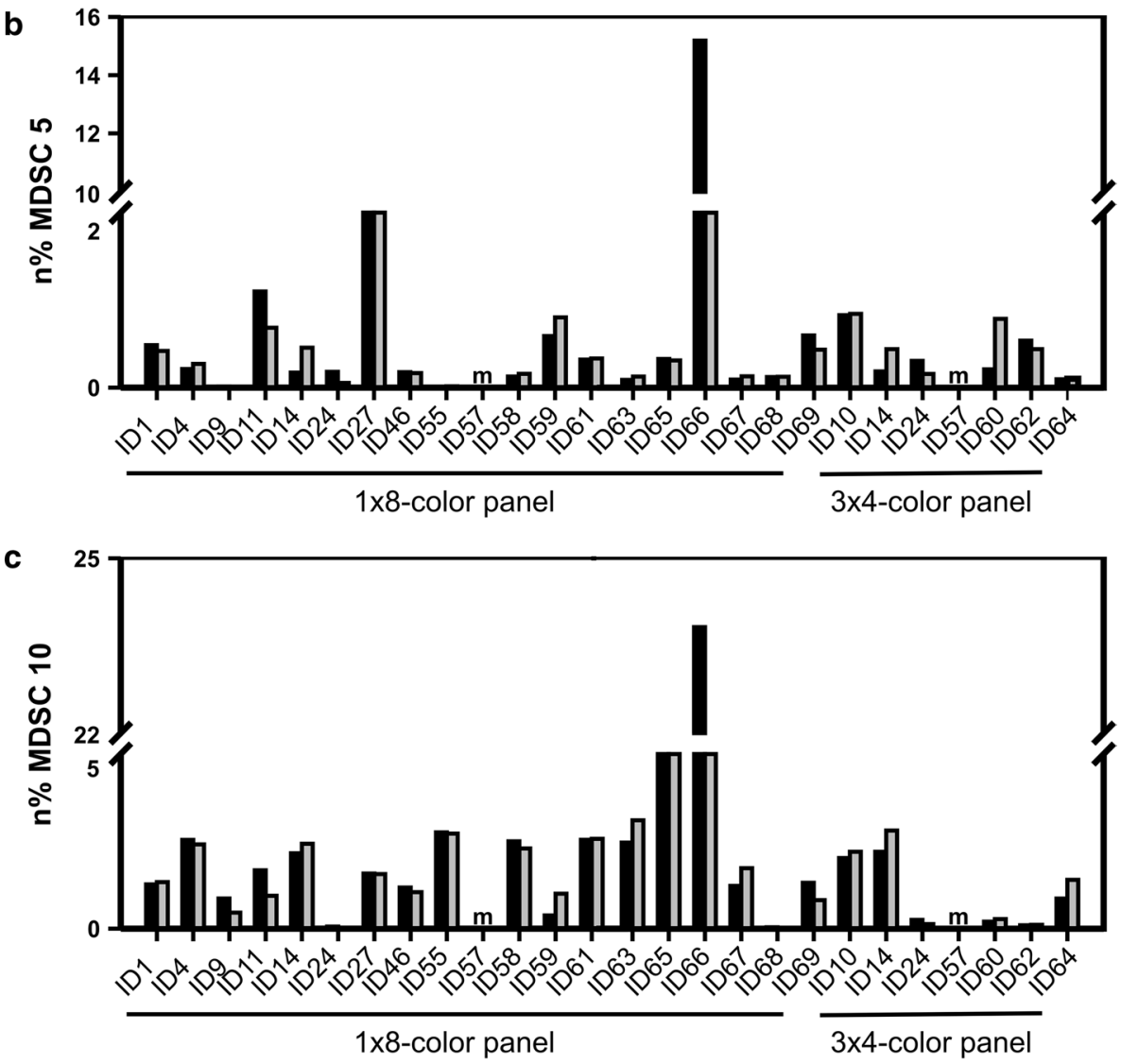

the validation of the predictive significance of MDSCs in multicenter studies is complicated by the phenotypic complexity of human MDSCs, thereby creating a challenge in finding a consensus on the minimal requirements for MDSC monitoring. To address this challenge, we organized the first proficiency panel to harmonize human MDSC phenotyping.

The task was challenging because of the large number of participants, the complexity of the staining panel and the number of subsets to be identified. Under these circumstances, it is not surprising that the quantification of the 10 requested MDSC subsets was characterized by a high inter-laboratory variance, increasing from monocytic to immature and granulocytic subsets. The number and the international origin of the participating laboratories indicate that the high inter-laboratory variance observed in the panel could be similar as in independent studies on human MDSC published. In contrast, the intra-laboratory variance was in general not high, indicating that the participating laboratories were experienced in the field of multicolor flow cytometry.

The choice of reagents and the gating strategy were critical parameters that impacted on the quantification of MDSC. The participating laboratories had been allowed 

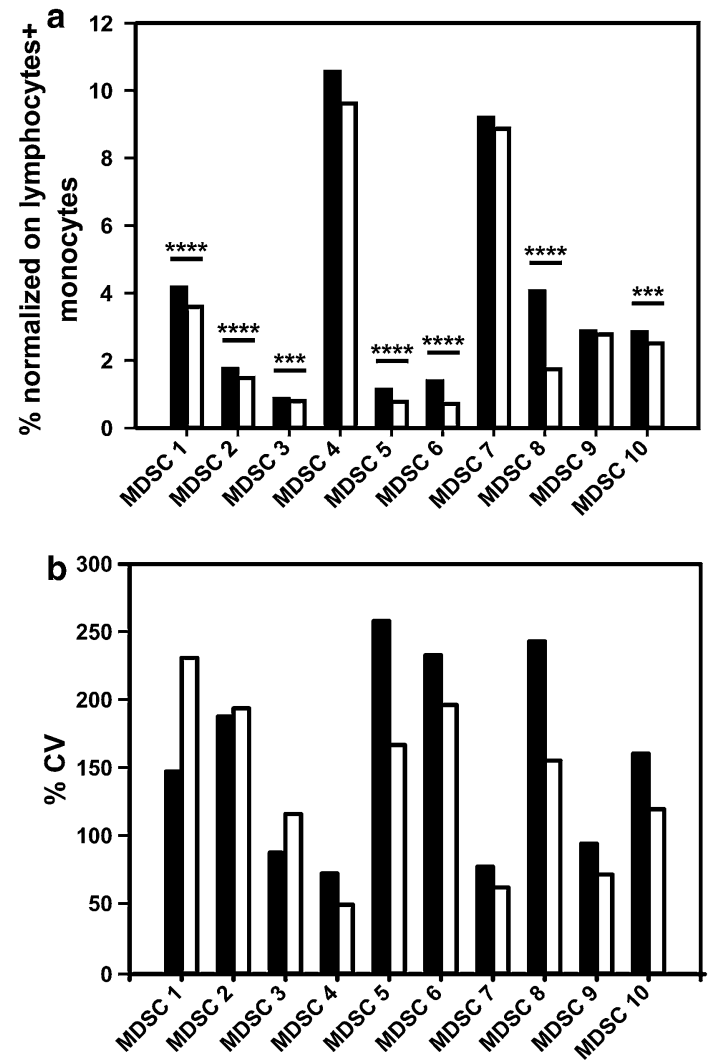

Fig. 4 a Average frequencies $(n=18$ evaluable laboratories reporting data for the 8-color panel) of MDSC subsets normalized on lymphocytes + monocytes $(n \%)$ identified either excluding or not the dead cells. Wilcoxon matched-pair test $(* * * P<0.001$, $* * * * P<0.0001)$. b Average \%CV $(n=18$ evaluable laboratories reporting data for the 8-color panel) of the frequencies of MDSC subsets identified either using a homogenous gating strategy (white bar) or not (black bars)

to decide on staining reagents. As a result, a considerable number of different antibodies were used to identify MDSC subsets using an 8-color staining panel. We observed that the clone of anti-CD124 antibody and the composition of the lineage cocktail significantly influenced the quantification of CD124 $4^{+}$MDSCs and immature MDSCs, respectively. The issue of the heterogeneity of reagents was also present in previous proficiency panels, in which a partial standardization of reagents had been suggested [25], especially for culturing of PBMCs intended to be used for functional $T$ cell assays [23, 26, 27]. The MDSC proficiency panel introduced the use of a DCM, and, as expected, we observed that the percentages of several MDSC subsets, and in particular of granulocytic ones, were significantly reduced by dead-cell exclusion. Hence, these results raise the question whether dead MDSCs should also be evaluated in frozen samples to account for their presence in fresh samples, or in contrast, whether inclusion of dead cells would alter MDSC quantification due to unspecific binding of antibodies to other cell types.

The gating strategies used for identification of MDSC subsets had a significant influence on the variance of results. Inter-laboratory performance improved significantly when analysis was limited to only those laboratories, which applied a homogenous gating strategy similar to that proposed in the panel's guidelines. This was not unexpected since most proficiency panels based on flow cytometry similarly recommend harmonization of the gating strategies across laboratories. In a multimer-based proficiency panel, results were audited in order to exclude wet laboratories which regularly used a wrong gating procedure [24, 25]. However, since identification of MDSC subsets derives from a complex combination of markers, we believe that a more adequate approach would be to train the participating laboratories to perform a properly gating pipeline through an in silico panel, as done in ICS proficiency panels [23, 28]. Hence, we are currently setting up an in silico panel to measure the influence of manual gating analysis on the variance of the results. In this study, participants will be asked to re-analyze their own data, as well as those of a supplementary reference dataset, following guided instructions for sequential gating. The output will guide us to design the second step of the proficiency panel in which harmonization should be achieved following a wider set of mandatory requests, dealing not only with gating procedure but also with other parameters such as the use of a DCM, a unique lineage cocktail, and fluorescence-minus-one controls for dimly fluorescent antigens.

\section{Conclusion}

Overall, this interim report emphasizes the lack of a robust definition for human MDSC subsets and highlights the importance of finding an agreement on the harmonized staining and analysis strategy and of translating our findings in general guidelines for immune phenotyping of these cells. We demonstrated that it is feasible to conduct a proficiency panel including a large number of participants and identifying a high number of myeloid subsets using 8-color flow cytometry. In this first step, we provided individual feedback of performance for each laboratory and we identified a number of parameters that must be harmonized in the second step, hence establishing the foundation for the development of a robust assay for MDSC phenotyping.

Acknowledgments The authors thank the participants of the proficiency panels, which are listed in the "Appendix." The CIMT Immunoguiding Program (CIP) is supported by the Wallace Coulter Foundation (FL, USA). Work in Padova was supported by grants from the Italian Ministry of Health and Italian Association for Cancer Research (AIRC Grant 12886 to Susanna Mandruzzato). Cécile Gouttefangeas 
is supported by a grant of the Forschungsgemeinschaft SFB685/Z05. We thank Sylvia Janetzki for co-organization in the USA.

\section{Compliance with ethical standards}

Conflict of interest The authors declare that they have no conflict of interest.

\section{Appendix: panel participants}

K. Bruderek, S. Brandau, University Hospital Essen, Otorhinolaryngology, Essen, Germany

I. Shevchenko, V. Umansky, Skin Cancer Unit, German Cancer Research Center, Heidelberg and Department of Dermatology, Venereology and Allergology, University Medical Center Mannheim, Ruprecht-Karl University of Heidelberg, Mannheim, Germany

C. Diaz, University of Miami, Miami, FL, USA

D. Riemann, B. Seliger, Martin Luther University Halle, Halle, Germany

A. Martens, H. Zelba, G. Pawelec, Center for Medical Research, University of Tübingen, Tübingen, Germany

M. Tazzari, A. Cova, C. Castelli and L. Rivoltini,

Unit of Immunotherapy of Human Tumors, Fondazione IRCCS Istituto Nazionale dei Tumori, Milan, Italy

R. Rae, S. Attig, Medizinische Klinik III, Universitätsmedizin Mainz, Mainz, Germany

D. Wycze, P. Rodri, A. Ochoa, Louisiana State University, New Orleans, LA, USA

A.R. Coleman, A. Cazaly, D. Joseph-Pietras, University of Southampton, Southampton, UK

J. Vella, P. Serafini, Dept. of Microbiology and Immunology, University of Miami, Miami, FL, USA

Y.P. De Coana, R. Kiessling, M. Nystrom, Karolinska Institute, Solna, Sweden

P. Rayman, Y. Yang, J. Finke, Cleveland Clinic, Cleveland, $\mathrm{OH}, \mathrm{USA}$

G. Winkels, K. Bublitz, Miltenyi Biotec, Bergisch Gladbach, Germany

S.R. Hadrup, M. Idorn, University Hospital Herlev, Herlev, Denmark

C. Alanio, M. Albert, L. Rogge, M. Hasan, Center for Human Immunology, Institut Pasteur, Paris, France

J. Hermes, I. Poschke, DKFZ, Heidelberg, Germany

T. Condamine, D. Gabrilovich, The Wistar Institute, Philadelphia, PA, USA

M.J.P. Welters, V.V. Visconti, J.J. Van Ham, S.H. van der

Burg, Leiden University, Leiden, The Netherlands

C. Ma, F. Korangy, T. Greten, NCI, Gastrointestinal Malignancy Section, Bethesda, MD, USA

J. Staats, J. Enzor, Duke CFAR Flow Core Facility, Durham, NC, USA
L.H. Butterfield, M.J. Buffo, University of Pittsburgh Cancer Institute Immunologic Monitoring Lab, Pittsburgh, PA, USA

V. Damuzzo, S. Mandruzzato, University of Padova, Padova, Italy

D. Maurer, S. Walter, Immatics Biotechnologies GmbH, Tübingen, Germany

Open Access This article is distributed under the terms of the Creative Commons Attribution 4.0 International License (http://creativecommons.org/licenses/by/4.0/), which permits unrestricted use, distribution, and reproduction in any medium, provided you give appropriate credit to the original author(s) and the source, provide a link to the Creative Commons license, and indicate if changes were made.

\section{References}

1. Gabrilovich DI, Ostrand-Rosenberg S, Bronte V (2012) Coordinated regulation of myeloid cells by tumours. Nat Rev Immunol 12(4):253-268. doi:10.1038/nri3175

2. Huang A, Zhang B, Wang B, Zhang F, Fan KX, Guo YJ (2013) Increased CD14(+)HLA-DR (-/low) myeloid-derived suppressor cells correlate with extrathoracic metastasis and poor response to chemotherapy in non-small cell lung cancer patients. Cancer Immunol Immunother 62(9):1439-1451. doi:10.1007/ s00262-013-1450-6

3. Solito S, Falisi E, Diaz-Montero CM, Doni A, Pinton L, Rosato A, Francescato S, Basso G, Zanovello P, Onicescu G, GarrettMayer E, Montero AJ, Bronte V, Mandruzzato S (2011) A human promyelocytic- like population is responsible for the immune suppression mediated by myeloid-derived suppressor cells. Blood 118(8):2254-2265. doi:10.1182/blood-2010-12-325753

4. Walter S, Weinschenk T, Stenzl A, Zdrojowy R, Pluzanska A, Szczylik C, Staehler M, Brugger W, Dietrich PY, Mendrzyk R, Hilf N, Schoor O, Fritsche J, Mahr A, Maurer D, Vass V, Trautwein C, Lewandrowski P, Flohr C, Pohla H, Stanczak JJ, Bronte V, Mandruzzato S, Biedermann T, Pawelec G, Derhovanessian E, Yamagishi H, Miki T, Hongo F, Takaha N, Hirakawa K, Tanaka H, Stevanovic S, Frisch J, Mayer-Mokler A, Kirner A, Rammensee HG, Reinhardt C, Singh-Jasuja H (2012) Multipeptide immune response to cancer vaccine IMA901 after single-dose cyclophosphamide associates with longer patient survival. Nat Med 18(8):1254-1261. doi:10.1038/nm.2883

5. Wang L, Chang EW, Wong SC, Ong SM, Chong DQ, Ling KL (2013) Increased myeloid-derived suppressor cells in gastric cancer correlate with cancer stage and plasma S100A8/A9 proinflammatory proteins. J Immunol 190(2):794-804. doi:10.4049/ jimmunol.1202088

6. Damuzzo V, Pinton L, Desantis G, Solito S, Marigo I, Bronte V, Mandruzzato S (2014) Complexity and challenges in defining myeloid-derived suppressor cells. Cytometry B Clin Cytom 88(2):77-91. doi:10.1002/cytob.21206

7. Solito S, Marigo I, Pinton L, Damuzzo V, Mandruzzato S, Bronte V (2014) Myeloid-derived suppressor cell heterogeneity in human cancers. Ann N Y Acad Sci 1319:47-65. doi:10.1111/nyas. 12469

8. Almand B, Clark JI, Nikitina E, van Beynen J, English NR, Knight SC, Carbone DP, Gabrilovich DI (2001) Increased production of immature myeloid cells in cancer patients: a mechanism of immunosuppression in cancer. J Immunol 166(1):678-689 
9. Kusmartsev S, Gabrilovich DI (2002) Immature myeloid cells and cancer-associated immune suppression. Cancer Immunol Immunother 51(6):293-298

10. Mirza N, Fishman M, Fricke I, Dunn M, Neuger AM, Frost TJ, Lush RM, Antonia S, Gabrilovich DI (2006) All-trans-retinoic acid improves differentiation of myeloid cells and immune response in cancer patients. Cancer Res 66(18):9299-9307. doi:10.1158/0008-5472.CAN-06-1690

11. Filipazzi P, Valenti R, Huber V, Pilla L, Canese P, Iero M, Castelli C, Mariani L, Parmiani G, Rivoltini L (2007) Identification of a new subset of myeloid suppressor cells in peripheral blood of melanoma patients with modulation by a granulocytemacrophage colony-stimulation factor-based antitumor vaccine. J Clin Oncol 25(18):2546-2553. doi:10.1200/JCO.2006.08.5829

12. Mandruzzato S, Solito S, Falisi E, Francescato S, Chiarion-Sileni V, Mocellin S, Zanon A, Rossi CR, Nitti D, Bronte V, Zanovello P (2009) IL4Ralpha + myeloid-derived suppressor cell expansion in cancer patients. J Immunol 182(10):6562-6568. doi:10.4049/jimmunol.0803831

13. Schmielau J, Finn OJ (2001) Activated granulocytes and granulocyte-derived hydrogen peroxide are the underlying mechanism of suppression of t-cell function in advanced cancer patients. Cancer Res 61(12):4756-4760

14. Brandau S, Trellakis S, Bruderek K, Schmaltz D, Steller G, Elian M, Suttmann H, Schenck M, Welling J, Zabel P, Lang S (2011) Myeloid-derived suppressor cells in the peripheral blood of cancer patients contain a subset of immature neutrophils with impaired migratory properties. J Leukoc Biol 89(2):311-317. doi:10.1189/jlb.0310162

15. Zea AH, Rodriguez PC, Atkins MB, Hernandez C, Signoretti S, Zabaleta J, McDermott D, Quiceno D, Youmans A, O'Neill A, Mier J, Ochoa AC (2005) Arginase-producing myeloid suppressor cells in renal cell carcinoma patients: a mechanism of tumor evasion. Cancer Res 65(8):3044-3048. doi:10.1158/0008-5472. CAN-04-4505

16. Rodriguez PC, Ernstoff MS, Hernandez C, Atkins M, Zabaleta J, Sierra R, Ochoa AC (2009) Arginase I- producing myeloidderived suppressor cells in renal cell carcinoma are a subpopulation of activated granulocytes. Cancer Res 69(4):1553-1560. doi:10.1158/0008-5472.CAN-08-1921

17. Diaz-Montero CM, Salem ML, Nishimura MI, Garrett-Mayer E, Cole DJ, Montero AJ (2009) Increased circulating myeloidderived suppressor cells correlate with clinical cancer stage, metastatic tumor burden, and doxorubicin-cyclophosphamide chemotherapy. Cancer Immunol Immunother 58(1):49-59. doi:10.1007/s00262-008-0523-4

18. Dumitru CA, Moses K, Trellakis S, Lang S, Brandau S (2012) Neutrophils and granulocytic myeloid- derived suppressor cells: immunophenotyping, cell biology and clinical relevance in human oncology. Cancer Immunol Immunother 61(8):11551167. doi:10.1007/s00262-012-1294-5

19. Kotsakis A, Harasymczuk M, Schilling B, Georgoulias V, Argiris A, Whiteside TL (2012) Myeloid-derived suppressor cell measurements in fresh and cryopreserved blood samples. J Immunol Methods 381(1-2):14-22. doi:10.1016/j.jim.2012.04.004
20. Kusmartsev S, Su Z, Heiser A, Dannull J, Eruslanov E, Kubler H, Yancey D, Dahm P, Vieweg J (2008) Reversal of myeloid cell-mediated immunosuppression in patients with metastatic renal cell carcinoma. Clin Cancer Res 14(24):8270-8278. doi:10.1158/1078-0432.CCR-08-0165

21. Trellakis S, Bruderek K, Hutte J, Elian M, Hoffmann TK, Lang S, Brandau S (2013) Granulocytic myeloid-derived suppressor cells are cryosensitive and their frequency does not correlate with serum concentrations of colony-stimulating factors in head and neck cancer. Innate Immun 19(3):328-336. doi: $10.1177 / 1753425912463618$

22. Lugli E, Roederer M, Cossarizza A (2010) Data analysis in flow cytometry: the future just started. Cytometry A 77(7):705-713. doi:10.1002/cyto.a.20901

23. Welters MJ, Gouttefangeas C, Ramwadhdoebe TH, Letsch A, Ottensmeier CH, Britten CM, van der Burg SH (2012) Harmonization of the intracellular cytokine staining assay. Cancer Immunol Immunother 61(7):967-978. doi:10.1007/ s00262-012-1282-9

24. Gouttefangeas C, Chan C, Attig S, Kollgaard TT, Rammensee HG, Stevanovic S, Wernet D, thor Straten P, Welters MJ, Ottensmeier C, van der Burg SH, Britten CM (2015) Data analysis as a source of variability of the HLA-peptide multimer assay: from manual gating to automated recognition of cell clusters. Cancer Immunol Immunother 64(5):585-598. doi:10.1007/ s00262-014-1649-1

25. Britten CM, Janetzki S, Ben-Porat L, Clay TM, Kalos M, Maecker H, Odunsi K, Pride M, Old L, Hoos A, Romero P (2009) Harmonization guidelines for HLA-peptide multimer assays derived from results of a large scale international proficiency panel of the Cancer Vaccine Consortium. Cancer Immunol Immunother 58(10):1701-1713. doi:10.1007/s00262-009-0681-Z

26. Chudley L, McCann KJ, Coleman A, Cazaly AM, Bidmon N, Britten CM, van der Burg SH, Gouttefangeas C, Jandus C, Laske K, Maurer D, Romero P, Schroder H, Stynenbosch LF, Walter S, Welters MJ, Ottensmeier CH (2014) Harmonisation of short-term in vitro culture for the expansion of antigen- specific CD8(+) T cells with detection by ELISPOT and HLA-multimer staining. Cancer Immunol Immunother 63(11):1199-1211. doi:10.1007/s00262-014-1593-0

27. Filbert H, Attig S, Bidmon N, Renard BY, Janetzki S, Sahin U, Welters MJ, Ottensmeier C, van der Burg SH, Gouttefangeas C, Britten CM (2013) Serum-free freezing media support high cell quality and excellent ELISPOT assay performance across a wide variety of different assay protocols. Cancer Immunol Immunother 62(4):615-627. doi:10.1007/s00262-012-1359-5

28. McNeil LK, Price L, Britten CM, Jaimes M, Maecker H, Odunsi K, Matsuzaki J, Staats JS, Thorpe J, Yuan J, Janetzki S (2013) A harmonized approach to intracellular cytokine staining gating: results from an international multiconsortia proficiency panel conducted by the cancer immunotherapy consortium (CIC/CRI). Cytometry A 83(8):728-738. doi:10.1002/cyto.a.22319 